\title{
Study of Multilayer Coatings of Ti/TiN/TiC Produced by Pulsed Arc Discharge
}

\author{
E. Restrepo, V. Benavides, A. Devia, S. Olarte, M. Arroyave, and Y.C. Arango \\ Laboratorio de Física del Plasma, Universidad Nacional de Colombia, Manizales, Colombia
}

Received on 3 February, 2004; revised version received on 25 May, 2004

\begin{abstract}
This work presents a study of multilayered Ti/TiN/TiC thin films obtained using a PAPVD (Plasma Assisted Physical Vapor Deposition) system by pulsed-arc discharge. For this purpose, a titanium target and a stainless steel substrate were used, placed on the cathode and on the anode, respectively, inside a vacuum chamber. To grow these films, different gases and concentrations were required. Ti is obtained with argon gas, TiN with nitrogen, and $\mathrm{TiC}$ with methane, at $2.5 \mathrm{mbar}$ and $5 \mathrm{~mm}$ distance between electrodes. By means of $\mathrm{X}$ ray diffraction (XRD), the phases present in the film were determined, observing (111), (200), and (100) orientations for both, TiN and TiC. Also, by employing XRD techniques, Titanium Atoms Distance (DTA) was calculated at the interface of TiN and TiC, in order to study the crystallographic match. Energy Dispersive Spectroscopy (EDS) was employed in order to carry out elemental analyses in the materials. These analyses were obtained for $12 \mathrm{keV}$ and $30 \mathrm{keV}$, observing the effects in the results. Taking advantage of defects generated during the growth of the multilayer, chemical composition maps were carried out, probing the combination of Ti and $\mathrm{N}$ in one layer and $\mathrm{Ti}$ and $\mathrm{C}$ in the other layer. Scanning Electron Microscopy (SEM) technique allowed observing the presence of the multilayer, as well as the measurement of the thicknesses of each layer, which are in the order of nanometers.
\end{abstract}

\section{Introduction}

Surface engineering of metallic substrates with ceramic coatings calls unanimous attention from industry and researchers, as it produces a host of properties such as wear and erosion resistance, and oxidation and corrosion resistance. However, a coating is highly functional only if the interface between the film and the substrate is sound and strong [1]. Transition metal nitrides, such as TiN, and carbides, such as TiC, have been widely used as hard protective coatings due to their excellent mechanical properties. There is, however, still an interest in coatings with further improved wear resistance, hardness, and fracture toughness over TiN and TiC. One of these methods consists in depositing them as multilayers. It was found that combining layers with different mechanical properties enhanced coating performance [2]. Multilayers are extensively used in semiconductor, optical, and metallurgy industries and are becoming of increasing importance in areas like magnetic recording, superconductors, and, lately, wear and corrosion protective coatings. Interest on PVD multilayers lies in their considerably enhanced micro-hardness [3]. Bromark reported that the concept of multilayered coatings offers a potent means to tailor the properties of tribological coatings and the metallic Ti can, for example, be used to control the coating residual stress state [4]. The mutual connection between the neighboring layers enables to obtain interfaces with different degrees of crystallographic match. One of the coatings which satisfy this condition best is the one based on TiN and TiC and their solutions [5]. These materials have been produced by using different techniques like laser ablation, [6] magnetron sputtering D.C. and R.F., [7] and vacuum arc, among others [8]. The cathodic vacuum arc is widely used to produce coatings. They generate an intense metallic plasma jet, with ion kinetic energies in the range of $20-100 \mathrm{eV}$, depending on the cathodic material. Among different coatings, of special interest are those obtained by combining metallic ions with atoms (or molecules) of reactive gas introduced into the discharge chamber [9]. Ti/TiN/TiC multilayers were grown using a PAPVD vacuum arc system. With the objective of understanding the properties of multilayered structures, different techniques were used.

\section{Experimental Details}

Ti/TiN/TiC multilayers were deposited on polished stainless steel (SS) by a vacuum arc system. The arc system consists of a vacuum chamber with two electrodes. The cathode is made up of a titanium target with $99.999 \%$ purity and the anode is a stainless steel 304 substrate. The discharge is generated by an $\mathrm{RLC}(\mathrm{R}=0.46 \Omega, \mathrm{L}=2.3 \mathrm{mH}, \mathrm{C}=54 \mathrm{mF})$ circuit in which the capacitor bank is charged until reaching the suitable voltage. The ignition of the discharge is performed by a high-voltage trigger source. Each film was deposited using different conditions. For the Ti layer, the chamber was filled with argon and a voltage of $300 \mathrm{~V}$ was used. With the TiN layer, the gas used is nitrogen and the voltage is also $300 \mathrm{~V}$. Finally, a TiC layer was obtained by filling the chamber with methane gas and using a voltage of $320 \mathrm{~V}$. 
TABLE 1. Data employed for calculation of average titanium atomic distance (DTA) in the interface.

\begin{tabular}{|c|c|c|c|c|c|}
\hline \multirow{2}{*}{ Material } & \multicolumn{5}{|c|}{ Interplanar Distance $\left(L_{h k l}\right)$} \\
\hline & 111 & 200 & 220 & 311 & 222 \\
\hline TiN & 2.44663 & 2.11884 & 1.49825 & 1.27771 & 1.22331 \\
\hline \multirow[t]{2}{*}{$\mathrm{TiC}$} & 2.49449 & 2.16029 & 1.52756 & 1.30271 & 1.24725 \\
\hline & \multicolumn{5}{|c|}{ Normalized Intensities $\left(I_{h k l}\right)$} \\
\hline TiN & 0.235 & 0.4535 & 1 & 0.528 & 0.3753 \\
\hline \multirow[t]{2}{*}{$\mathrm{TiC}$} & 0.1513 & 1 & 0.7094 & 0.4678 & 0.3863 \\
\hline & \multicolumn{5}{|l|}{$\alpha_{h k l}$} \\
\hline TiN & 0.0906 & 0.1749 & 0.3858 & 0.2037 & 0.1448 \\
\hline $\mathrm{TiC}$ & 0.0557 & 0.3683 & 0.2613 & 0.1723 & 0.1423 \\
\hline
\end{tabular}

All the coatings were produced at 2.5 mbar pressure and $5 \mathrm{~mm}$ distance between electrodes. This system has been described in details in previous works $[10,11]$. For XRay characterization we employed a Bruker AXS diffractometer, model D8 Advance, geometry of parallel beams with graphite monochromator. SEM micrographs were taken with Philips ESEM XL- 30 TPM equipment, allowing for magnifications between $6 \mathrm{X}$ and $1200000 \mathrm{X}$, with detector for EDS analysis.

\section{Results and Discussion}

$\mathrm{X}$ - ray diffraction pattern for the $\mathrm{Ti} / \mathrm{TiN} / \mathrm{TiC}$ multilayer is shown in Fig. 1. It was possible to observe peaks (111), (200) and (100) corresponding to FCC structures of TiN and TiC. The fact that both coatings have the same crystallographic orientation could be explained by the fact that these two materials, considered as refractory transition metal nitride and carbide, have similar characteristics. It should be mentioned that none of these compounds have the ideal rock- salt structure, assumed by theory, but they deviate from the stoichiometry. Moreover, it is found, in the case of these two materials that all three main types of bonding contribute to their refraction properties: metallic contribution with a finite density of states at the Fermi energy, $E f$, ionic bonding caused by transfer electrons from the metal to the non-metal atom, and covalent bonds consisting mainly of strong interaction between non-metal $p$ and metal $d$ states, but also on metal/metal interactions [12]. Lattice parameters in both materials were also measured, with the following findings: for TiN $4.23768 \pm 0.00125 \AA$ and for $\mathrm{TiC}$ $4.32058 \pm 0.00171 \AA$.

On the basis of the results presented in Table 1, the percentage of crystallographic planes $(h k l)$ in the TiN and TiC were calculated. See eq. (1).

$$
\alpha_{h k l}^{T i N}=\frac{I_{111}^{T i N}}{I_{111}^{T i N}+I_{200}^{T i N}+I_{220}^{T i N}+I_{311}^{T i N}+I_{222}^{T i N}}
$$

where $\alpha_{111}^{T i N}$ is the percentage of (111) crystallographic planes in TiN and $I_{111}^{T i N}$ the spectral line intensity for (111) crystallographic plane in TiN.

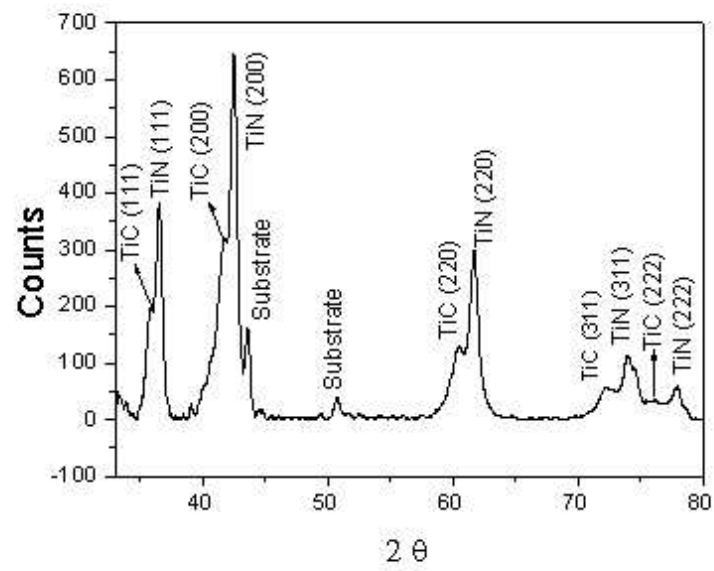

Figure 1. XRD pattern of Ti/TiN/TiC multilayered film.

Considering the lattice parameters and the analysis of the TiN atoms position in the different crystallographic planes of FCC, authors calculated the average titanium atoms distance $\left(L_{T i N}\right)$ in TiN and $\left(L_{T i C}\right)$ in TiC:

$$
\begin{gathered}
L_{T i N}=\alpha_{111}^{T i N} L_{111}^{T i N}+\alpha_{200}^{T i N} L_{200}^{T i N}+\alpha_{220}^{T i N} L_{220}^{T i N} \\
+\alpha_{311}^{T i N} L_{311}^{T i N}+\alpha_{222}^{T i N} L_{222}^{T i N} \\
L_{T i C}=\alpha_{111}^{T i C} L_{111}^{T i C}+\alpha_{200}^{T i C} L_{200}^{T i C}+\alpha_{220}^{T i C} L_{220}^{T i C} \\
+\alpha_{311}^{T i C} L_{311}^{T i C}+\alpha_{222}^{T i C} L_{222}^{T i C}
\end{gathered}
$$

Applying these results the authors have calculated the differences in the average titanium atoms distance (DTA) for layers being in contact with the $\mathrm{TiN}$ and $\mathrm{TiC}$ interface. $L_{111}^{T i N}$ and $L_{111}^{T i C}$ are the atoms distance in the (111) crystallographic plane for TiN and $\mathrm{TiC}$ respectively. 


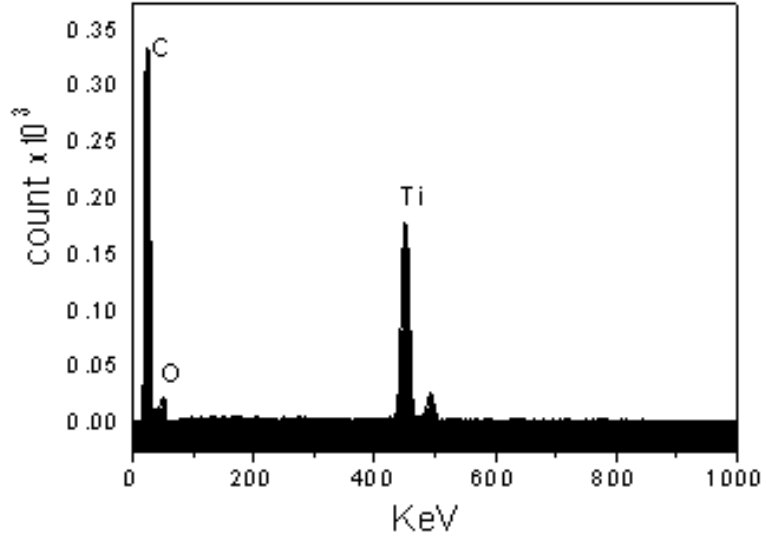

(a)

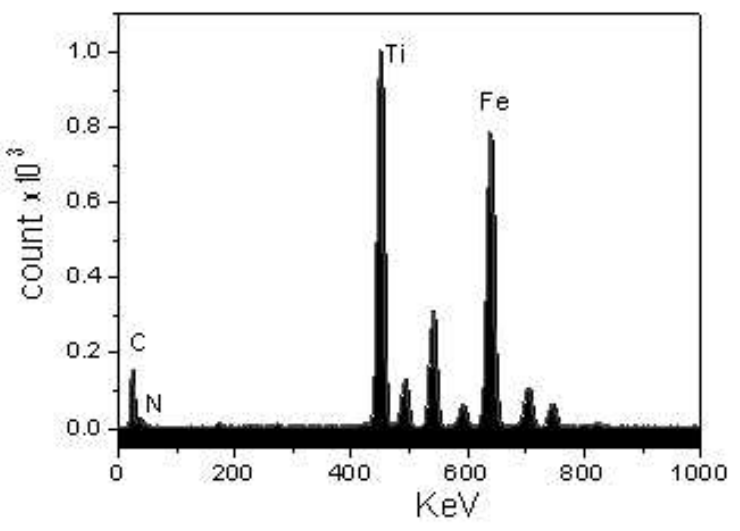

(b)

Figure 2. EDS spectra of the multilayer (a) with electron beam energy of $12 \mathrm{KeV}$. (b) with electron beam energy of $30 \mathrm{KeV}$.

The values obtained are $L_{T i N}=1.6076 \AA$ and $L_{T i C}=$ $1.7356 \AA$

$$
D T A_{T i C-T i N}=L_{T i C}-L_{T i N}=0.128 \AA
$$

where $D T A_{T i N-T i C}$ is the difference in the average titanium atoms distance for layers being in contact in the interface. It is observed that this value is lower than the lattice parameter of $\mathrm{TiN}$ and $\mathrm{TiC}$, showing that there is a match in the interlayer. This method is widely explained by J. Smolik et al [13].

Figure 2 shows various EDS spectra taken with different electron beam energy. The spectrum presented in Fig. 2(a) was obtained with $12 \mathrm{keV}$. Lines of Ti, C, and $\mathrm{O}$ were identified, (the last possibly due to the contamination of the film when it is exposed to the environment). The spectrum of Fig. 2(b) was taken at $30 \mathrm{keV}$ of electron beam energy. In this case we observed $\mathrm{Ti}, \mathrm{C}, \mathrm{N}, \mathrm{O}$, and $\mathrm{Fe}$. The elemental composition of the spectra changes with the beam energy because in the first case, the energy is not high enough to allow the production of characteristic X-ray in the TiN layer. In the second case, the high energy lets the technique get information on all the layers and the substrate. Another way to observe the multilayer composition is to use chemical composition maps obtained by using EDS. Taking advantage of defects generated when the multilayer was grown, it was

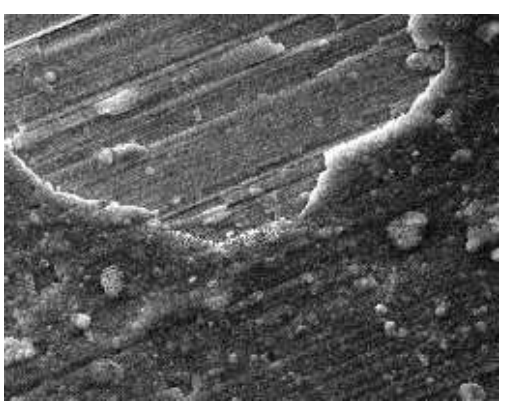

(a)

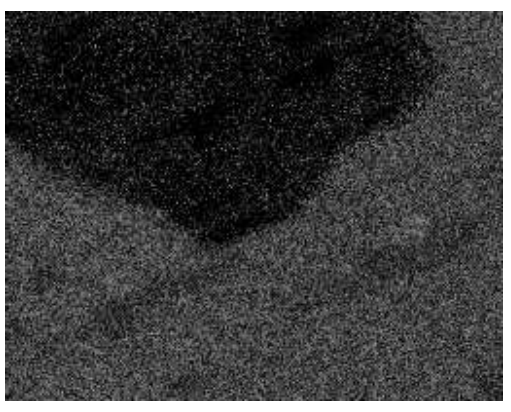

(b)

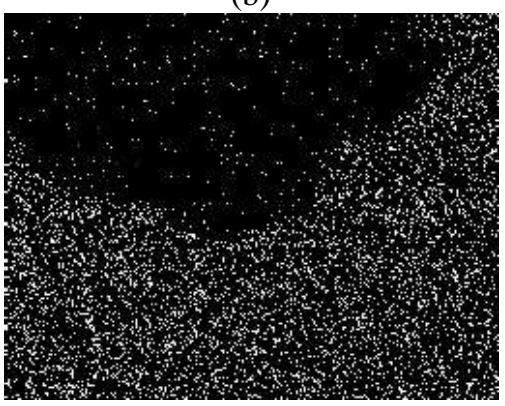

(c)

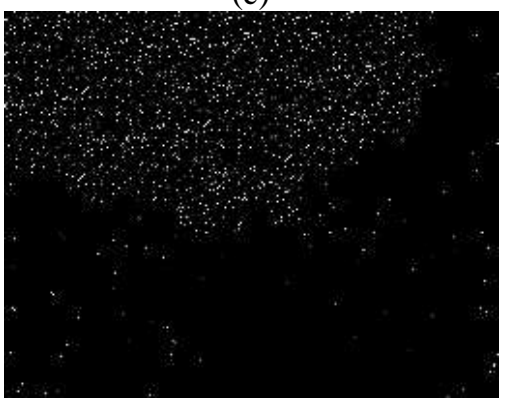

(d)

Figure 3. (a) SEM micrograph using a defect of the multilayer, (b) Chemical map elemental composition for Ti, (c) Chemical map elemental composition for $\mathrm{C}$, (d) Chemical map elemental composition for N. E. Restrepo et. al.

possible to compare the elemental composition on the TiN and TiC layer. Figs. 3(a)-(d) show these maps. Fig. 3(a) shows a SEM micrograph of the multilayer, observing both, TiN and TiC coatings. Fig. 3(b) presents a chemical map realized for Ti material; the contrast is due to the different 
chemical concentration for each layer. Figs. 3(c) and 3(d) represent the chemical map for $\mathrm{N}$ and $\mathrm{C}$ respectively. The lighter zones in both cases show the concentration of these elements. Fig. 4 shows SEM images of a cross-section of the multilayer, which measured the thickness of each layer. The results are $48 \mathrm{~nm}$ for Ti, $66.3 \mathrm{~nm}$ for TiN, and $66.3 \mathrm{~nm}$ for TiC, observing that these films are very thin.

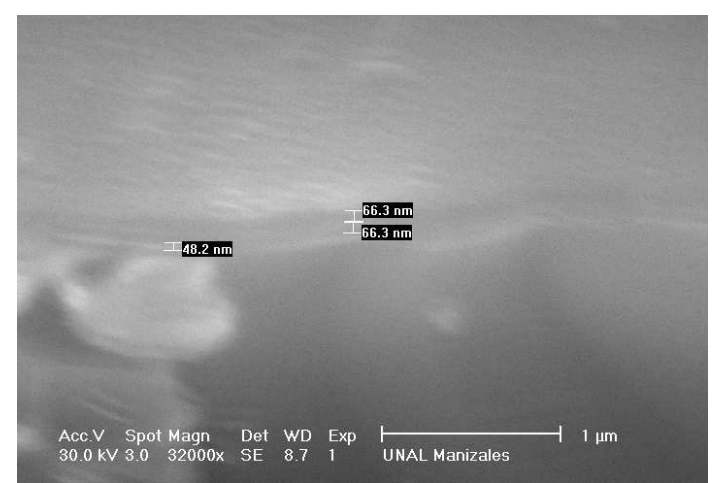

Figure 4. SEM micrograph employed to measure the thickness of the films.

\section{Conclusions}

Through XRD, EDS, and SEM a series of Ti/TiN/TiC multilayers, grown by plasma assisted pulsed-arc discharge, were studied. An agreement was achieved between the chemical composition analysis carried out with different techniques. It was found by using XRD that the TiN and TiC films have the same orientation and crystalline structure, indicating a possible good match between them, due to their similar refraction conditions. The elemental chemical maps obtained for the multilayer allowed demonstrating the existence of all the elements that were expected in this case and by using different values of electron beam energy, we corroborated that the composition of the film changes as a function of depth.

The thickness of the film was measured by using SEM, obtaining values in the order of nanometers.

\section{Acknowledgments}

The author gratefully acknowledge the finantial support of the Division para la Investigación de la Universidad Nacional de Manizales (DIMA) and el Instituto Colombiano para el Desarrollo de la Ciencia y la Tecnología (Colciencias) during the course of this research, under project RC292-2001

\section{References}

[1] L. R. Katipelli, A. Agarwal, and N. B. Dahotre, Mat. Sci. Eng. A 289, 34 (2000).

[2] U. Helmersson, S. Todovora, S. A. Barnett, J. S. Sundgren. H. Markett, and J. E. Greene, J. Appl. Phys. 62, 481 (1987).

[3] C. J. Tabares, L. Rebouta, B. Almeida, J. Bessa e Sousa, M. F. da Silva, and J. C. Soares, Thin Solid Films 317, 124 (1998).

[4] T. S. Li, H. Li, and F. Pan, Surf. Coat. Technol. 137, 225 (2001).

[5] J. Smolik, K. Zdunek, Vacuum 55, 147 (1999). .

[6] G. Radhakrishnan, P. M. Adams, and D. M. Speckman, Thin Solid Films 358, 131 (2000).

[7] J. Wang, W. Z. Li, and H. D. Li, Thin Solid Films 382, 190 (2001).

[8] M. H. Shiao, F. S. Shieu, Thin Solid Films 386, 27 (2001).

[9] A. Lepone, H. Kelly, and A. Marquez, J. Appl Phys. 90, 3174 (2001).

[10] E. Restrepo and A. Devia, J. Vac Sci. technol. A 22, 377 (2004).

[11] E. Restrepo, B. Segura, M. Arroyave, and A. Devia, Revista Colombiana de Física, 34, 493 (2002).

[12] K. Schwarz and A. Neckel, Phys. Status Solidi B, 225, 265 (2001).

[13] J. Smolik, K. Zdunek, Vacuum, 55, 147 (1999). 